\title{
1) \\ Directed Functionalization of Quinoxalines Aiming the Synthesis of Bioactive Compounds
}

\author{
Samuel R. A. Ferreira*, Jesus M. Pena, Simone C. Silva, Giuliano C. Clososki \\ Research Center for Natural and Synthetic Products - FCFRP - University of São Paulo \\ *samuelremotto@hotmail.com
}

Keywords: directed metalation, organometallic compounds, functionalized quinoxalines.

\section{INTRODUCTION}

Access to functionalized organometallic compounds has increased considerably the scope of these nucleophilic reagents in organic synthesis. Directed metalation using mixed lithium-magnesium bases as TMPMgCl.LiCl are widely used for a smooth metalations of several aromatics and heterocycles with a good functional group compatibility. ${ }^{1}$ However, deprotonation of substituted quinoxalines gave unsatisfactory results due to the high reactivity of the intermediate magnesium species. ${ }^{2}$ Herein, we wish to report a direct method for the deprotonation and functionalization of some substituted quinoxalines by using $\mathrm{TMPMgCl} . \mathrm{LiCl}$ in the presence of $\mathrm{ZnCl}_{2}$.

\section{RESULTS AND DISCUSSION}

An approach described by Harms has afforded 2quinoxalinecarboxylic 1 in reasonable yield. From this compound we have achieved the preparation of ester and amides derivatives in good yields. Some examples are shown in Scheme 1.

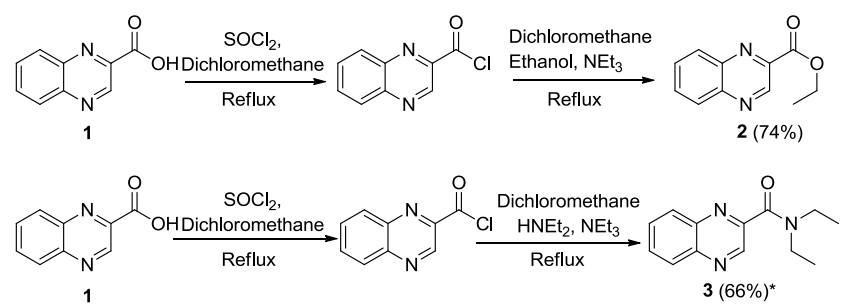

Scheme 1

A variety of functionalized quinoxalines were prepared through the reaction of the substrates with TMPMgCl. $\mathrm{LiCl}$ in the presence of $\mathrm{ZnCl} 2$ followed by the reaction with electrophiles. We can consider that first the base TMPMgCl.LiCl reacts with heterocycle affording the organomagnesium intermediate and then a fast transmetalation with $\mathrm{Zn}$ occurs, leading to the quinoxalilzinc intermediate (4), which is more stable than organomagnesium intermediate (Scheme 2).

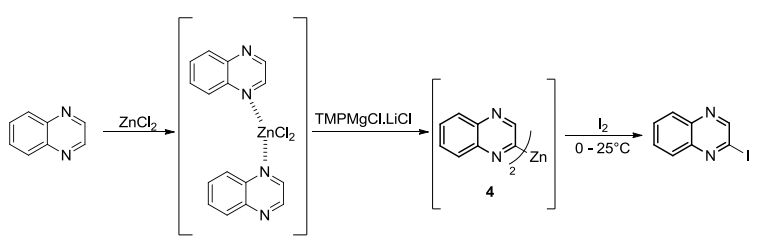

Scheme 2

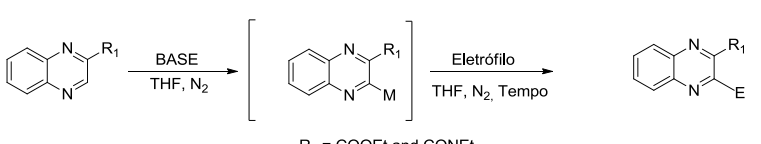

$\mathrm{R}_{1}=$ COOEt and CONEt $\mathrm{t}_{2}$

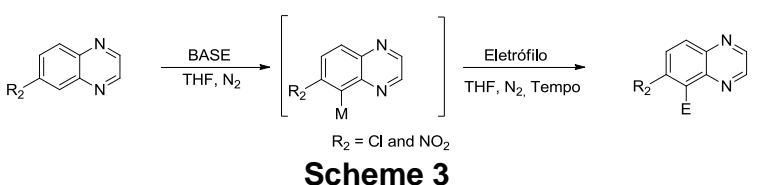

Some examples of functionalized quinoxalines prepared by the methodology described above are shown below:
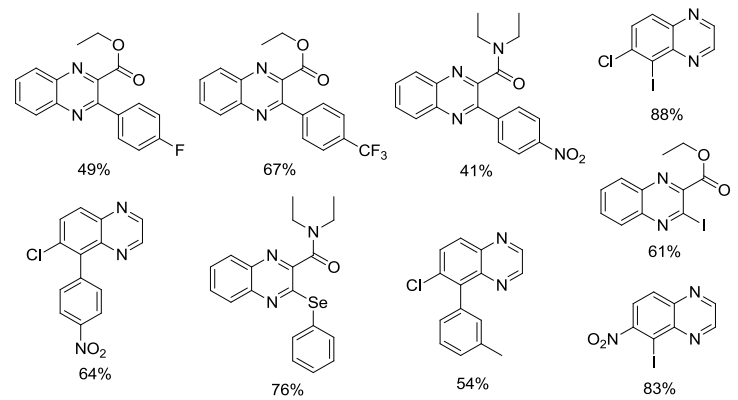

Figure 1. Products obtained by direct metalation of the substrates with $\mathrm{TMPMgCl}$. $\mathrm{LiCl}$ in the presence of $\mathrm{ZnCl}_{2}$.

\section{CONCLUSION}

In summary, metalation of substituted quinoxalines with $\mathrm{TMPMgCl} . \mathrm{LiCl}$ in the presence of $\mathrm{ZnCl}_{2}$ has allowed the synthesis of highly functionalized derivatives in modest to good yields. The scope of the methodology and the application to the synthesis of bioactive compound is under investigation in our laboratories.

\section{ACKNOWLEDGEMENTS}

FAPESP, FCFRP-USP, NPPNS, CAPES and Cnpq

\section{REFERENCES}

${ }^{1}$ Wanderlich, S. H., Rohbogner, C. J., Unsinn, A., Knochel, P. Organic Process Research and Development. 2010, 339, 345.

2 Dong, Z. Clososki, G.C. Wunderlich, S. H. Unsinn, A. Li, Jinshan. Knochel, P. Chem. Eur. J. 2009, 15, 457. 